\title{
Improving Soliton Compression Quality with Cascaded Nonlinearities by Engineered Multi-section Quasi-phase-matching Design
}

\author{
Zeng, Xianglong; Guo, Hairun; Zhou, Binbin; Bache, Morten
}

Published in:

CLEO Technical Digest

Publication date:

2012

Document Version

Publisher's PDF, also known as Version of record

Link back to DTU Orbit

Citation (APA):

Zeng, X., Guo, H., Zhou, B., \& Bache, M. (2012). Improving Soliton Compression Quality with Cascaded Nonlinearities by Engineered Multi-section Quasi-phase-matching Design. In CLEO Technical Digest (pp. JW4A.60). Optical Society of America.

\section{General rights}

Copyright and moral rights for the publications made accessible in the public portal are retained by the authors and/or other copyright owners and it is a condition of accessing publications that users recognise and abide by the legal requirements associated with these rights.

- Users may download and print one copy of any publication from the public portal for the purpose of private study or research.

- You may not further distribute the material or use it for any profit-making activity or commercial gain

- You may freely distribute the URL identifying the publication in the public portal 


\title{
Improving Soliton Compression Quality with Cascaded Nonlinearities by Engineered Multi-section Quasi-phase-matching Design
}

\author{
Xianglong Zeng ${ }^{1,2}$, Hairun Guo ${ }^{1}$, BinBin Zhou ${ }^{1}$, Morten Bache ${ }^{1}$ \\ ${ }^{1}$ DTU Fotonik, Department of Photonics Engineering, Technical University of Denmark, 2800 Lyngby, Denmark. \\ ${ }^{2}$ The Key Lab of Specialty Fiber Optics and Optical Access Network, Shanghai University, 200072 Shanghai, China. \\ xzen@fotonik.dtu.dk
}

\begin{abstract}
In few-cycle soliton generation with large compression factors using cascaded nonlinearities the pulse quality can be improved by engineering quasi-phase-matching structures. The soliton-induced mid-IR optical Cherenkov wave is also enhanced.

(C) 2011 Optical Society of America

OCIS codes: $(190.5530,320.5520,190.7110)$
\end{abstract}

Generation of high-quality optical pulses as short as few optical cycles from solid-state lasers and fiber amplifiers with hundreds of femtosecond durations has been the fascinating tool used as the stable and compact optical sources for ultrafast spectroscopy, pump-probing and biology. So far, few-cycle pulses have been generated in the near-IR wavelength region by compressing pulses in gas-filled cells or hollow capillaries to compress higher energy pulses, but they suffer from being hard to control and very bulky [1]. An alternative is to use solitons from self-defocusing cascaded nonlinearities (e.g., phase-mismatched second-harmonic generation, SHG): when they are strong enough to overcome the cubic (Kerr) self-focusing nonlinearity, few-cycle solitons can be generated in the visible and nearIR without beam filamentation or break-up [2]. Recently a temporal nonlocal model gave insight in optimal phasemismatch ranges where few-cycle pulse durations are possible [3] and this led to an experimental demonstration of 17 fs compressed solitons (less than 4 optical cycles) from 47 fs near-IR pulses through cascaded quadratic soliton compression (CQSC) in a short standard lithium niobate crystal [4]. However, the soliton compression mechanism has similar drawbacks to higher-order soliton compression: the larger the soliton number, the larger the unwanted pedestal, and the less energy remains in the compressed spike. Such features are detrimental to practical applications, and is in particular a problem when compressing longer $>>100$ fs pulses to sub-20 fs duration.

Here we address this problem by using quasi-phase matching (QPM) with a multi-section design. The effective self-defocusing nonlinear strength gives an effective soliton number $N_{\text {eff }}^{2}=L_{d} \frac{\omega_{1}}{c} I_{\mathrm{FW}}\left(\left|n_{\text {casc }}^{I}\right|-n_{\text {Kerr }}^{I}\right)$, where $n_{\text {casc }}^{I} \propto$ $-d_{\text {eff }}^{2} / \Delta k_{\mathrm{QPM}}$ is the Kerr-like cascading nonlinearity, controlled by the quadratic nonlinearity $d_{\text {eff }}$ and the QPM phase mismatch $\Delta k_{\mathrm{QPM}}$, and $n_{\text {Kerr }}^{I}$ is the self-focusing material Kerr nonlinearity. The fundamental wave (FW) dispersion length is $L_{d} \equiv T_{\mathrm{FW}, \text { in }}^{2} /\left|k_{F}^{(2)} W\right|, T_{\mathrm{FW} \text {,in }}$ is the $\mathrm{FW}$ input duration and $k_{\mathrm{FW}}^{(2)}=d^{2} k_{\mathrm{FW}} / d \omega^{2}$ is the $\mathrm{FW}$ GVD parameter. The basic idea is that for low $N_{\text {eff }}$ a small pedestal and high pulse quality can be realized [5], so higher-order soliton compression should be possible in multi-section QPM structure where each section maintains a low effective soliton order. This is done by engineering the QPM domain length $\Lambda_{j}$ so $n_{\text {casc }}^{I}$ is changed by altering the residual QPM phase mismatch $\Delta k_{j \text {,eff }}=\Delta k-2 \pi / \Lambda_{j}$, where $\Delta k=k_{2}-2 k_{1}$ is the material phase mismatch. Each section is designed so at the end the soliton is compressed to duration $\tau_{j}$, for which the soliton roughly experiences a unity soliton order. In the following section $N_{j \text {,eff }}$ is then adjusted to be larger than unity by choosing a new lower $\Lambda_{j}$ value. As we show now, it turns out to work remarkably well already as a two-section and three-section design.

We consider $5 \% \mathrm{MgO}$ doped periodically poled lithium niobate for pulse compression at the wavelength of $1.56 \mu \mathrm{m}$, where high-energy Er-doped fiber amplifiers operate. We use type-0 phase-mismatched SHG for soliton compression because of its large quadratic nonlinear component $d_{\mathrm{eff}}=d_{33}$, and we used $n_{\mathrm{Kerr}}^{I}=17 \cdot 10^{-20} \mathrm{~m}^{2} / \mathrm{W}$. The full coupled SHG propagation equations under the slowly-evolving-wave approximation including exact dispersion, self-steepening and Kerr effects are solved numerically [5]. Fig. 1(a-b) show the comparison of the compressed output in one- and twosection QPM structures. It is clearly shown that the compressed pulse is quite clean and the peak intensity is almost doubled while reaching the limit of pulse compression (less than 3 optical cycles) in two-section QPM, which consists of two sequential gratings with the length $L_{1}\left(L_{2}\right)=0.21(0.012) L_{d}$, the total length is $47 \mathrm{~mm}$ (dispersion length $\left.L_{d}=211 \mathrm{~mm}\right)$ and the input soliton number of $N_{\text {eff }}=5\left(I_{1, \text { in }}=96 \mathrm{GW} / \mathrm{cm}^{2}\right)$. Fig.1 (c) shows that soliton compression in three-section QPM with length of $L_{1}: L_{2}: L_{3}=0.78: 0.07: 0.01$. The quality factor $\left(Q_{c}\right)$ of the compressed pulse, defined as the ratio of sech $^{2}$-fitted energy of the pulse central spike over the input pulse energy, is as high as 0.75 due to 

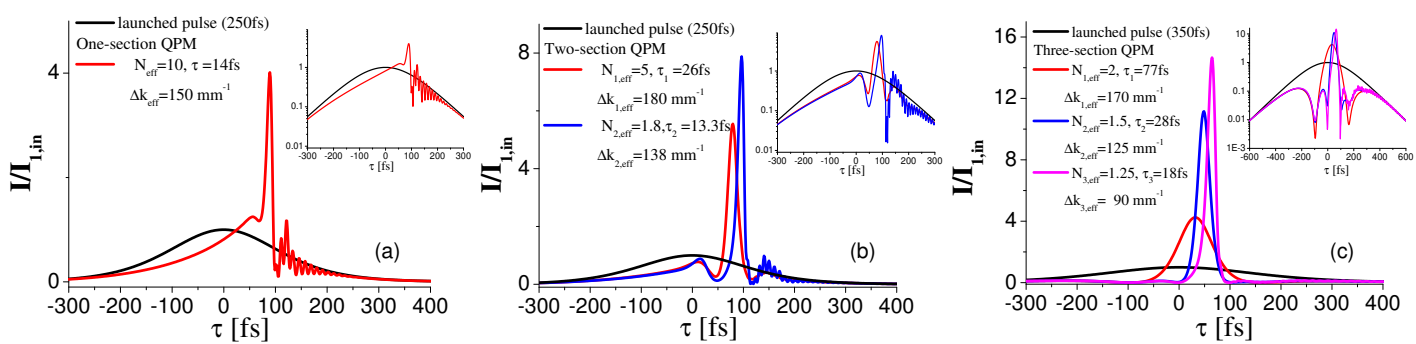

Fig. 1. Numerical simulations of compressed pulses in multi-section QPM structures of pump wavelength $1.56 \mu \mathrm{m}$. Less than 3 cycle pulses compressed from 250 fs FWHM launched pulses in (a) one-section QPM : $L=0.09 L_{d}$ with input soliton number of $N_{\text {eff }}=10\left(96 \mathrm{GW} / \mathrm{cm}^{2}\right)$ and (b) two-section QPM of total length $47 \mathrm{~mm}: L_{1}\left(L_{2}\right)=0.21(0.012) L_{d}$ with input soliton number of $N_{\text {eff }}=5$; (b) 350 fs FWHM launched pulses in three-section QPM with input soliton number of $N_{\mathrm{eff}}=2\left(5.7 \mathrm{GW} / \mathrm{cm}^{2}\right)$. The insets show the logarithmic scales.

the smaller pedestal. Fig.2 (a-b) show the the quality factor and the optimum pulse duration versus the effective soliton

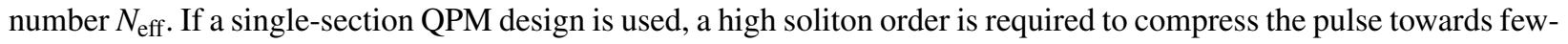
cycle duration, and this leads to a reduced $Q_{c}[5]$ as well as increased group-velocity mismatch induced self-steepening. In addition, the large soliton order leads to reduced pulse compression due to competing cross-phase-modulation effects [5]. In contrast, the multi-section QPM design, strong pulse compression towards few-cycle duration is possible while keeping a high pulse quality because the soliton order is kept low.

The broadband mid-IR accompanied with CQSC stems from soliton-induced optical Cherenkov radiation [6], and it was found to be greatly enhanced by using multi-section QPM as shown in Fig.2 (c). As $\Delta k_{\text {eff }}$ decreases in the sequential QPM and the peak intensity increases, strong trapping between fundamental and second harmonic components occur, which leads to the slower group velocity of soliton. From the spectrum evolution of fundamental wave, it is clear that more energy from soliton is transferred to the Cherenkov radiation around the wavelength of $3 \mu \mathrm{m}$ in the second QPM. Multi-section QPM is therefore advantageous for efficient generation of few-cycle mid-IR waves.
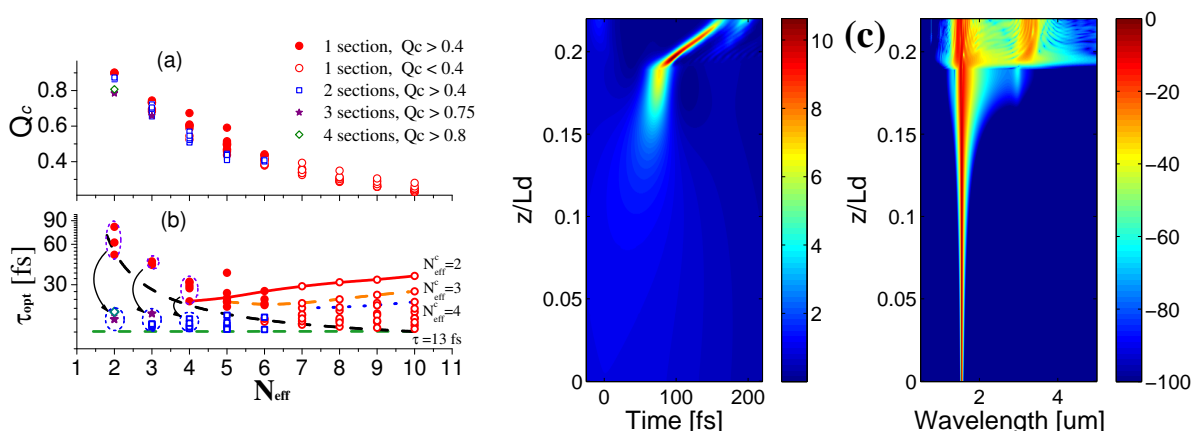

Fig. 2. Results of numerical simulations showing the optimum compression parameters versus $N_{\text {eff }}$ in multi-section gratings for (a) quality factor, (b) the minimum pulse duration. The filled mark with round symbols are the compression results in one section QPM with good quality factor $\left(Q_{c}>0.4\right)$ and the square, star and diamond symbols represents the results in multi-section QPM with high quality factor while durations reaching the limit of 13 fs. (c) FW temporal and spectral evolution in two-section QPM.

Concluding, we showed a new scheme for compressing longer $>>100 \mathrm{fs}$ pulses through a soliton effect to few-cycle duration with high quality. This was achieved in a multi-section engineered QPM structure exploiting controllable cascaded nonlinearities, where the soliton order in each section was kept low. Additionally the soliton-induced mid-IR Cherenkov wave was greatly enhanced in the process.

\section{References}

1. M. Nisoli, S. De Silvestri, and O. Svelto, "Generation of high energy $10 \mathrm{fs}$ pulses by a new pulse compression technique," Appl. Phys. Lett. 68, 2793-2795 (1996)

2. F. Wise and J. Moses, "Self-focusing and self-defocusing of femtosecond pulses with cascaded quadratic nonlinearities," in "Self-focusing: Past and Present,", vol. 114 of Topics in Applied Physics, R. Boyd et al. eds. (Springer Berlin / Heidelberg, 2009), pp. 481-506.

3. M. Bache, O. Bang, J. Moses, and F. W. Wise, "Nonlocal explanation of stationary and nonstationary regimes in cascaded soliton pulse compression," Opt. Lett. 32, 2490-2492 (2007).

4. B.B. Zhou, A. Chong, F.W. Wise and M. Bache, "Few-cycle solitons in short strongly phase-mismatched frequency conversion crystals,"submitted, arXiv: 1109.4261 .

5. M. Bache, J. Moses, and F. W. Wise, "Scaling laws for soliton pulse compression by cascaded quadratic nonlinearities," J. Opt. Soc. Am. B 24, 2752-2762 (2007). [erratum: ibid., 27, 2505 (2010)].

6. M. Bache, O. Bang, B.B. Zhou, J. Moses, and F. Wise, "Optical Cherenkov radiation by cascaded nonlinear interaction: an efficient source of energetic few-cycle near- to mid-IR pulses,” Opt. Express 19, 22557-22562 (2011). 